\title{
Percepción competencial del emprendimiento en personas con discapacidad. Un estudio comparativo
}

\author{
Angel José Olaz Capitán \\ Pilar Ortiz García \\ Universidad de Murcia
}

Resumen

El propósito de este trabajo es identificar a través de la comparativa entre dos colectivos: personas con discapacidad que desarrollan actividades de emprendimiento y el de técnicos de la Administración Regional relacionados con este ámbito qué dimensiones y aspectos competenciales favorecen y / o limitan la actividad emprendedora de las personas con algún tipo de discapacidad física y/o sensorial. Desde una perspectiva metodológica cualitativa y en base a muestras estructurales, el análisis se desarrolló a través de dos herramientas: la Técnica Nominal de Grupo para identificar dimensiones de análisis y el test Emotional Competence Intelligence para ponderar qué competencias psicosociales eran claves en la comprensión del fenómeno. Tras el análisis en cada uno de estos colectivos este trabajo concluye estableciendo las oportunas conclusiones comparativas.

Palabras clave

Autoconocimiento personal; Competencias de autogestión; Conciencia social; Deficiencia; Gestión de relaciones; Técnica de Grupo Nominal

Códigos JEL: J15, J24, L26, 015

\section{COMPETENCIAL PERCEPTION OF ENTREPRENEURSHIP IN PEOPLE WITH DISABILITIES. A COMPARATIVE STUDY}

\section{Abstract}

The aim of this work is to identify through the comparison between two groups: people with disabilities who develop entrepreneurship activities and technicians of the Regional Administration related to this area which dimensions and competencies favour and / or limit the entrepreneurial activity of people with some kind of physical and / or sensory disability. From a qualitative methodological perspective and based on structural samples, the analysis was developed through two tools: the Nominal Group Technique to identify dimensions of analysis and the Emotional Competence Intelligence test to assess which psychosocial competences were key in understanding the phenomenon. After the analysis in each of these groups this work concludes establishing the appropriate comparative conclusions.

\section{Keywords}

Self-knowledge; Self-management Competencies; Social conscience; Deficiency; Relations Management; Nominal Group Technique

JEL codes: J15, J24, L26, 015

Fecha de recepción del original: 25 de abril de 2018; versión definitiva: 5 de febrero de 2019 .

Angel José Olaz Capitán, Universidad de Murcia. Departamento de Sociología. Facultad de Economía y Empresa. Campus Universitario de Espinardo. 30100 Espinardo (Murcia)

Tel.: +34 868884118; E-mail: olazcapi@um.es. ORCID ID:0000-0001-8285-8227

Pilar Ortiz García, Universidad de Murcia. Departamento de Sociología. Facultad de Economía y Empresa. Campus Universitario de Espinardo. 30100 Espinardo (Murcia)

Tel.: +34 868883853; E-mail: portizg@um.es. ORCID ID: 0000-0001-7679-0772 


\title{
Percepción competencial del emprendimiento en personas con discapacidad. Un estudio comparativo ${ }^{1}$
}

\author{
Angel José Olaz Capitán \\ Pilar Ortiz García \\ Universidad de Murcia
}

1. Introducción

Este trabajo pretende realizar una aproximación al fenómeno del emprendimiento en aquellas personas con discapacidad física y sensorial a través de un estudio en clave competencial.

En concreto, el estudio identifica las dimensiones y aspectos competenciales que propician y/o limitan la actividad emprendedora de este colectivo, a través del análisis realizado a personas con discapacidad que desarrollan actividades emprendedoras y el de aquellos técnicos de la Administración Regional implicados en este ámbito de actividad.

Debido a las dificultades formales que supone precisar conceptualmente el término discapacidad, este trabajo comenzará por delimitarlo, abordando a continuación, el emprendimiento en este colectivo, sin olvidar el significado del término competencia.

Tras resolver estas cuestiones terminológicas este artículo se detendrá en los aspectos metodológicos: la Técnica de Grupo Nominal (en adelante, T.G.N.) y el test Emotional Competence Intelligence (E.C.I.), merced a los cuales se identificaron, tanto las dimensiones, como las competencias que vehiculizan y moderan el proceso de emprendimiento en personas con discapacidad visual, auditiva y/o motórica.

\footnotetext{
1 La investigación se desarrolla al amparo del Proyecto Discapacidad y Emprendimiento. Análisis Competencial (CSO201675818R) convocatoria para el año 2016 correspondiente al Programa Estatal de Fomento de la Investigación Científica y Técnica de Excelencia, Subprograma Estatal de Generación de Conocimiento, en el marco del Plan Estatal de Investigación Científica y Técnica y de Innovación 2013-2016 financiado por el Ministerio de Economía y Competitividad del Gobierno de España.
}

Finalmente, este artículo prosigue con el análisis de las diferentes percepciones que se tienen del emprendimiento en ambos colectivos, examinado en su caso las coincidencias y discrepancias de este proceso desde la perspectiva del emprendimiento en personas con discapacidad

\section{Algunas cuestiones terminológicas previas}

Según la Clasificación Internacional de Deficiencias, Discapacidades y Minusvalías (CIDDM) deben distinguirse varios y distintos aspectos (OMS, 1997) aunque estrechamente relacionados:

- La enfermedad entendida en el sentido más genérico de su expresión que comprende cualquier tipo de enfermedad, trastorno o accidente. La enfermedad está recogida por la Organización Mundial de la Salud (OMS) en su Clasificación Internacional de Enfermedades y Problemas de Salud Relacionados (CIE).

- La deficiencia que es la representación o exteriorización directa de las consecuencias de la enfermedad, manifestándose tanto en los órganos del cuerpo como en sus funciones (también se incluyen las psicológicas).

- La discapacidad hace referencia al modo en que se $o b$ jetiva la deficiencia en la persona, con la consiguiente repercusión directa en su capacidad para la realización de actividades en los términos considerados normales para 
cualquier individuo de sus características por razón de edad, género, etc. Es, por tanto, una restricción o ausencia de lo considerado como normal.

- La minusvalía se refiere a la "socialización" de la problemática causada en una persona por las consecuencias derivadas de una enfermedad, manifestada a través de la deficiencia y/o la discapacidad, y que afecta al desempeño del papel social que le es propio. Este concepto lleva implícito un componente sociocultural que ayuda en su formulación y comprensión.

Esta diferenciación resulta clave para delimitar distintos conceptos que, aun estando íntimamente relacionados, presentan matices diferentes.

Según el Real Decreto 1971/1999, de 23 de diciembre, por el que se determina el procedimiento para el reconocimiento, declaración y calificación del grado de minusvalía, las discapacidades pueden clasificarse en tres grandes grupos:

- Las discapacidades físicas ${ }^{2}$ que se encuentran vinculadas con el cuerpo, miembros y órganos. Se originan en los sistemas músculo-esquelético, nervioso, respiratorio, cardiovascular, hematopoyético, digestivo, aparato urogenital, endrocrino, piel, anejos y neoplasias.

- Las discapacidades sensoriales ${ }^{3}$ asociadas al aparato visual, oído, garganta y todas aquellas estructuras relacionadas con el lenguaje.

- Las discapacidades psíquicas relacionadas con el retraso y/o enfermedad mental.

En el estudio realizado y dado las particulares características que concurren en el emprendimiento, esto es, cierto grado de iniciativa, autonomía y continuidad las personas con discapacidad psíquica no han sido contempladas en esta fase del proyecto de investigación al requerir de un acompañamiento por parte de otra persona que vulnerara los principios rectores antes formulados. Por otra parte, los resultados de la utilización del cuestionario $\mathrm{ECl}$ sobre personas con discapacidad psíquica, no serían comparables con los obtenidos sobre personas que no presentan este tipo de discapacidad, ya que una

\footnotetext{
2 Las discapacidades físicas, son de tipo motórico y/o visceral. Fundamentalmente son las siguientes: Sistema músculo esquelético (deficiencias articulares, amputaciones...); Sistema nervioso (epilepsia, tetraplejia, paraplejia...); Aparato respiratorio (asma, trasplante de pulmón, fibrosis quística...); Sistema cardiovascular (cardiopatías, arritmias...); Sistema hematopoyético y sistema inmunitario (anemias, Inmunodeficiencias no secundarias a infección por VIH...); Aparato digestivo (enfermedad del hígado, incontinencia...); Aparato genitourinario (deficiencias del riñón, incontinencia urinaria...); Sistema endocrino (hipertiroidismo, diabetes...); Piel y anejos (soriasis...) y Neoplasias (tumores)

${ }^{3}$ Las discapacidades sensoriales, entre las que se mencionan las auditivas y las visuales. En el terreno de la discapacidad sensorial auditiva. La Discapacidad sensoria auditiva recoge la carencia, disminución o deficiencia de la capacidad auditiva parcial o total. Dependiendo del momento en que esta se haya producido, el itinerario educativo y/o rehabilitador, pueden encontrarse personas que realizan la lectura labio-facial y que puedan comunicarse oralmente, hasta otras cuya comunicación se hace a través del lenguaje de signos. La Discapacidad sensorial visual se refiere a la carencia o afectación del canal visual de adquisición de la información. Se observan diferentes grados de limitación la ceguera y la baja visión.
}

parte de su contenido se refiere a competencias de carácter psicológico.

\section{El emprendimiento en las personas con discapacidad}

A pesar de los avances sociales de las últimas décadas, la integración de las personas con discapacidad sigue siendo una cuestión pendiente. Mejorar la empleabilidad de este colectivo es un reto social de primera magnitud. Entre los objetivos enunciados por programas tales como Europa 2020 de la Comisión Europea, figura la consecución de sociedades inclusivas. En este sentido, una de las primeras medidas es conocer los distintos problemas a los que se enfrentan colectivos en riesgo de exclusión social para diseñar políticas que la combatan en cualquiera de sus formas. Las personas con discapacidad son uno de los colectivos más expuestos a situaciones de vulnerabilidad.

Dada la amplitud del concepto y la variada casuística que recoge, resulta oportuno aclarar lo que se entenderá por discapacidad, concepto establecido por la Clasificación Internacional del Funcionamiento, de la Discapacidad y de la Salud, que define la discapacidad como un término genérico que engloba deficiencias, limitaciones de actividad y restricciones para la participación de la persona en actividades consideradas normales para cualquier individuo de sus características. La discapacidad denota los aspectos negativos de la interacción entre personas con un problema de salud (como parálisis cerebral, síndrome de Down o depresión) y factores personales y ambientales (como actitudes negativas, transporte y edificios públicos inaccesibles, y falta de apoyo social). Así se pone de manifiesto en el Informe Mundial de Discapacidad de la Organización Mundial de la Salud (OMS, 2001).

Según este Informe, el colectivo de personas con discapacidad supone más de mil millones en el mundo (un 15\% de la población mundial según las estimaciones de 2010 recogidas en la Encuesta Mundial de Salud). En España, la información que se recoge en la base Estatal del Instituto de Mayores y Servicios Sociales (IMSERSO), es la relativa al histórico de las personas que han solicitado el reconocimiento de la situación de discapacidad, así como el resultado de las valoraciones efectuadas por los equipos de evaluación de las distintas comunidades autónomas, incluidas las ciudades autónomas de Ceuta y Melilla (con información actualizada a 31 de diciembre de 2015). Del resultado de este proceso de valoración se obtiene un total de 2.998.639 personas con discapacidad, es decir, con un grado de discapacidad reconocido igual o mayor al 33\%, de acuerdo con lo que establece el artículo 4.2 del Real Decreto Legislativo 1/2013, de 29 de noviembre, por el que se aprueba el Texto Refundido de la Ley General de derechos de las Personas con Discapacidad y de su Inclusión Social.

Atendiendo a las tipologías, las discapacidades se distribu- 
yen de la siguiente forma: osteoarticular, $(28,0 \%)$, enfermedad crónica (18,0\%); mental (16,1\%); neuro-muscular (10,1\%); intelectual (9,0\%); visual (7,0\%); auditiva (5,8\%); mixta (2,7\%); expresiva $(0,5 \%)$ y un porcentaje residual correspondiente a las categorías "otras" y "no consta".

Estas cifras se mantienen al alza debido a varios factores: por una parte, la evolución demográfica en la que se observa un proceso cada vez más intenso del envejecimiento. Por otro, los modos de vida, que han producido un incremento global de enfermedades crónicas (tales como problemas cardiovasculares, trastornos mentales, diabetes, etc.), sin olvidar otros derivados de factores ambientales y también sociales.

Estos datos sitúan a este colectivo en una situación de vulnerabilidad importante, dada la mayor probabilidad de caer en situaciones de exclusión social. Una de las formas más importantes de inclusión es el trabajo, la consecución de un empleo y su desempeño. El trabajo constituye una de las formas más eficaces de proporcionar cohesión social, a la vez que independencia, seguridad económica y sentido de pertenencia social para individuo.

La información sobre inserción laboral de personas con discapacidad aparece recogida en diversas fuentes. En España se han realizado tres macro-encuestas por parte del Instituto $\mathrm{Na}$ cional de Estadística (INE) en 1986, 1999 y 2008: la Encuesta sobre Discapacidades, Deficiencias y Minusvalías (EDDM1986), la Encuesta sobre Discapacidades, Deficiencias y Estado de Salud (EDDS1999) y la última Encuesta de Discapacidad, Autonomía personal y situaciones de Dependencia (EDAD2008). No obstante, el contenido de estas encuestas, siendo esencial para el conocimiento del colectivo, contiene datos muy básicos sobre actividad económica y, en ningún caso sobre intención o materialización de algún tipo de actividad emprendedora. Dicha información sí aparece en la encuesta "El Empleo de las personas con discapacidad" del INE, aunque con un limitado nivel de desagregación y detalle.

Este vacío parcial se pone de manifiesto en la producción científica sobre el tema, que es tratado de forma transversal en diferentes disciplinas (Sociología, Psicología, Medicina, etc.), sin que en ellas se acabe consolidando una especialidad propiamente reconocida. La Sociología da cuenta de una reseñable producción científica desde finales de los años ochenta. En España cabe mencionar los estudios de Jiménez Lara (1991; 1992; 1998a y b; 2001; 2002; 2005; 2007); Casado (1991); Jiménez y Huete (2002a y b, 2003); Rodríguez Cabrero (2002); Rodríguez y Pérez (2004); De Lorenzo (2003); Zarco y García de la Cruz (2004); Pérez Bueno (2004); Fernández Enríquez (2005); Díaz Casanova (1985); Bascones (2006); Romañach (2002); Romañach y Palacios (2007) y los de Vázquez Ferreira (2005, 2007a y b, 2008, 2010 y 2011), entre otros.

No obstante, esta producción queda lejos de la generada sobre este tema en la tradición investigadora anglosajona. Desde dicha tradición se parte de la hipótesis que lleva a concebir la discapacidad como una construcción social que estigmatiza a las personas con algún tipo de limitación de sus capacidades físicas o psíquicas. Esta idea lleva a que las políticas activas dirigidas a la inclusión de personas con discapacidad se introduzcan en la agenda social, ya que se trata de una cuestión que afecta a todos.
La discapacidad es un fenómeno multidimensional que afecta a la persona desde una perspectiva biológica, psicológica y social. De todas ellas, la dimensión social ha sido una de las menos estudiadas (Vázquez Ferreira, 2008). Sin embargo, la determinación de los factores sociales sobre los biológicos y psicológicos es clara, de ahí la oportunidad de estudios que propicien actuaciones encaminadas a la normalización e integración plena de las personas con discapacidad en los entronos en los que interactúan.

El entorno laboral resulta clave para conseguir la normalización a la que se ha hecho referencia y, en particular, las experiencias emprendedoras. Conocer las posibilidades de las personas con discapacidad a partir del diagnóstico de competencias que las hacen especiales para acometer empresas de valor social, ecológico y humano, se convierte en un objetivo fundamental de cualquier sociedad que aspire a alcanzar la calificación de "inclusiva".

Algunas fuentes como el observatorio Global Entrepreneurship Monitor (GEM), constituye una de las principales bases de datos internacional sobre la actividad emprendedora. Sin embargo, no incluye un apartado específico que permita el seguimiento de dicha acción en personas con discapacidad.

De todo ello se desprende que, si bien se está avanzando en la recopilación de información y se dispone de fuentes estadísticas y estudios sobre el tema de discapacidad y emprendimiento, no resultan suficientes y, sobre todo, no son lo suficientemente específicas sobre esta cuestión.

\section{Los aspectos competenciales}

Antes de profundizar en los aspectos metodológicos es oportuno hacer una breve referencia al significado del término competencia.

McClelland (1973) define inicialmente el término competencia como algo que realmente causa un rendimiento superior en el trabajo, anteponiendo su significado y dimensión al enfoque educativo para ponerlo en correspondencia con otros elementos alternativos como el género, etnia o clase social para medir el rendimiento laboral de la persona en el contexto organizativo.

Desde entonces muchos han sido los posteriores desarrollos que se han realizado tomando como referencia esta primera definición. Casi de forma coincidente en el tiempo Bloom (1975) hace referencia a las competencias, aunque desde la óptica educativa, al mencionar que la "enseñanza basada en competencias", se asienta en cinco grandes postulados: Todo aprendizaje es esencialmente individual; el individuo, al igual que cualquier sistema, se orienta hacia la consecución de un conjunto de metas a lograr; el proceso de aprendizaje es más fácil cuando el individuo sabe qué es exactamente lo que 
se espera de él (algo no tan sencillo como pudiera parecer); el conocimiento preciso de los resultados contribuye a facilita el proceso aprendizaje y, es más probable que un alumno haga lo que se espera de él y lo que él mismo desea, si tiene la responsabilidad en las tareas de aprendizaje.

Los principios enumerados por Bloom se convierten tiempo después en la referencia, a partir de la cual se construyeron los modelos de educación y formación basados en competencias tanto en Estados Unidos como en Gran Bretaña.

Fue precisamente en este último país, donde el modelo fue aceptado, consolidándose hasta hoy en día. La competencia era entendida como el resultado necesario de la formación (Tuxworth, 1989).

Gracias a los originales puntos de partida que tanto McClelland como Bloom han presentado, este es el momento en el que se hace necesario observar las diferentes evoluciones del concepto competencia desde tres puntos de vista no necesariamente contrapuestos sino complementarios.

En suma, un conjunto de matizaciones orientadas a una mejor definición del término competencia que tiempo más tarde no pasan inadvertidas a Lawler (1994) a quien se le debe su aplicabilidad práctica al mundo empresarial o cómo las ventajas de gestionar por competencias, implica el paso de la burocratización a un modelo sistémico-organizativo.

En nuestro país, los trabajos realizados por Pereda y Berrocal (2001) inspirados en las ideas de Le Boterf, Barzucchetti y Vincent (1993) describen cinco elementos que, en su opinión, ayudan a definir la naturaleza de la competencia. Estos elementos se corresponderían con cinco tipos de saberes: saber o conocimientos que posee la persona y que le permitirán llevar a cabo los comportamientos incluidos en la competencia; saber hacer o la capacidad que tiene esa persona para aplicar aquellos conocimientos orientados a la solución de problemas o conflictos; saber estar o la realización de esos comportamientos en función de los procedimientos propios de la organización; querer hacer o querer llevar a cabo los comportamientos que articulan a la competencia, lo que alude directamente a la motivación del individuo y, finalmente, poder hacero las características que permiten al individuo disponer de los medios y recursos pertinentes necesarios para desarrollar su competencia.

Ya más recientes en el tiempo, son los estudios de naturaleza epistemo-metodológica de De Haro (2004) que, en un intento por clasificar la infinidad de modelos -en opinión de éste, tantos como autores-, se han ido sucediendo en el transcurso del tiempo. En este sentido, la propuesta que realiza se basa en la comprensión del término competencia, según sea entendida como variable dependiente o independiente.

Es en este segundo caso, al ser caracterizada como variable independiente, la competencia es contemplada como causa u origen del desempeño o resultado. A su vez, las causas que determinan el desempeño eficaz y eficiente de los trabajadores son de varios tipos: las que se basan en rasgos, en conductas o en una combinación de ambos.

De este modo, concluye señalando que la competencia es un constructo con el que se califican comportamientos relacionados entre sí, siendo éstos los responsables directos de un resultado excelente en el desempeño del puesto de trabajo.

\section{Cuestiones Metodológicas}

Los aspectos metodológicos de este trabajo motivan el enfatizar en las dos herramientas empleadas en su desarrollo: la Técnica de Grupo Nominaly el test Emotional Competence Intelligence (se denominará E.C.I.).

Merced a estos dos instrumentos, se definieron en primer lugar las dimensiones o ámbitos de estudio del emprendimiento en personas con discapacidad a través de la realización de las TT.GG.NN. desarrolladas con personas emprendedoras con discapacidad y técnicos de la Administración Pública y, sobre esa base, se sometieron a discusión cuáles eran las competencias clave moderadoras del emprendimiento a través del cuestionario E.C.I.

Como resultado de esta metodología de trabajo se detectaron qué competencias incidían en el emprendimiento de las personas con discapacidad, según ámbitos de estudio y colectivos representados. Las siguientes líneas explicarán las características de ambos elementos y su imbricación metodológica

\subsection{La T.G.N. y definición de dimensiones de análisis}

Sobre el modelo original descrito para la técnica de Van de Ven y Delbecq, varios son los elementos que han sido variados en este proyecto de investigación, sin por ello querer faltar a sus objetivos principales como son: identificar y enriquecer la comprensión acerca del problema a través de un discurso susceptible de cuantificación; establecer un conjunto de hipótesis relacionadas con el significado y efectos del problema investigado y centrar la atención en las principales áreas o escenarios de investigación (Van de Ven y Delbecq, 1972: 338).

Tampoco se ha querido renunciar a sus objetivos, esto es, identificar las dimensiones críticas de un problema por medio de un proceso grupal no amenazante y despersonalizado; aclarar el significado de los puntos críticos, tanto para investigadores como para los participantes a través de la acción discursiva; explorar objetiva y subjetivamente las dimensiones que envuelven al problema objeto de estudio; identificar los pesos explicativos a través de procedimientos de votación (en este caso debería hablarse mejor de establecimiento de una prelación) y contribuir al desarrollo de hipótesis y la formulación de la redacción de preguntas orientadas a la investigación y entrevistas en posteriores trabajos de campo. (Van de Ven y Delbecq, 1972: 341).

Bajo estos supuestos, Gutiérrez (2011: 117), en referencia a las técnicas de investigación cualitativas, tanto su inconsistencia técnica como la aparente simpleza de su aplicación práctica, sugiere la utilización de prácticas artesanales no to- 
Cuadro 1. Fases comparadas de los modelos

\begin{tabular}{|c|c|c|c|}
\hline Fase & Adaptación metodológica & Fase & T.G.N. clásica \\
\hline Fase 1 & Introducción & Fase 1 & Introducción \\
\hline Fase 2 & Generación silenciosa de ideas & Fase 2 & Generación silenciosa de ideas \\
\hline Fase 3 & Agrupación de personas & Fase 3 & Listado Round-Robin \\
\hline Fase 4 & Discusión en grupo & Fase 4 & Discusión en grupo \\
\hline Fase 5 & Selección y jerarquización tarjetas & & \\
\hline Fase 6 & Colocación de las tarjetas & & \\
\hline \multirow[t]{5}{*}{ Fase 7} & Pausa o coffee break & Fase 5 & Pausa o coffee break \\
\hline & & Fase 6 & Listado priorizado de elementos "problemáticos" \\
\hline & & Fase 7 & Discusión y votación \\
\hline & & Fase 8 & Reactualización del Ranking de prioridades \\
\hline & & Fase 9 & Conclusión \\
\hline Fase 8 & Discusión seriada de las ideas plasmadas & & \\
\hline Fase 9 & Cuantificación del peso de los escenarios & & \\
\hline Fase 10 & Desarrollo de un plan de acción & & \\
\hline Fase 11 & Asociación de competencias a planes de acción & & \\
\hline Fase 12 & $\begin{array}{l}\text { Constitución de los Equipos Autónomos de Traba- } \\
\text { jo (EE.AA.TT.) }\end{array}$ & & \\
\hline
\end{tabular}

Fuente: elaboración propia.

talmente formalizadas y/o estandarizadas, extremadamente singulares y virtuosas, bien sea en referencia a los procesos desarrollados como a los productos alcanzados que al final están muy condicionadas a la figura del investigador. Estas cuestiones explicarían las adaptaciones metodológicas que se realizan, por ejemplo, a nivel muestral ${ }^{4}$ buscando una mejora en la gestión del tiempo de sesión (Dobbie et al. 2004: 402-406) y de tantos otros investigadores que quizás por no despertar críticas silencien sus adaptaciones procedimentales. Estas cuestiones unidas a la reflexión de Ruiz (2012: 160) que evitaría hablar de técnicas cualitativas y más de prácticas cualitativas, dado el carácter abierto y flexible de los procedimientos por los que se aborda la investigación social cualitativa, ayudarían a comprender la adaptación de la T.G.N. a nuestro estudio.

En el intento por establecer un diálogo entre el modelo establecido por Delbecq y Van De Ven (1971) y Delbecq, Van de Ven y Gustafson (1975) y la adaptación del modelo realizado, el Cuadro 1 recoge la sucesión de etapas que jalonan ambos esquemas.

En síntesis, la adaptación metodológica sustituye la votación del modelo original, tras la discusión de ideas, por el trabajo en microgrupos, consensuando las dimensiones que deterioran o limitan el desarrollo del emprendimiento y potenciando el desarrollo de recomendaciones que permitan revertir tales situaciones, además de razonar en términos de competencias.

\footnotetext{
${ }^{4}$ Este estudio ha empleado muestras estructurales que como es sabido está especialmente indicada Este tipo de muestreo está especialmente indicado para acceder a poblaciones de difícil registro o localización.
}

\subsection{El E.C.I. y elección de competencias}

El Emotional Competence Intelligence (E.C.I.) ha mostrado la validez del constructo en lo referente a la intuición y dimensiones de pensamiento-sentimiento (introversión y extraversión) (Burckle, 2000) y ha sido empleado a la hora de correlacionarlo con el clima laboral (Sala, 2003) y creencias irracionales de los individuos (Welpe et al., 2005).

Por último, dos estudios examinaron la validez discriminante del $\mathrm{ECl}$ con respecto al pensamiento crítico (Byrne, 2003), concluyendo la idoneidad en la arquitectura del constructo.

En el estudio sobre emprendimiento y discapacidad, tras la definición de las dimensiones o ámbitos de estudio a través de la T.G.N., se sometió a los diferentes colectivos seleccionados a que identificaran las competencias clave moderadoras del emprendimiento a través del E.C.I.

El modelo empleado en la investigación supone una adaptación de esta herramienta al fenómeno del emprendimiento. El modelo identifica cuatro grandes dimensiones (Autoconocimiento Personal, Autogestión, Conciencia Social y Gestión de Relaciones) que contienen un total de 18 aspectos competenciales; dichos aspectos son captados a partir de 18 cuestiones cada una de las cuales se traslada a una escala Likert de 4 niveles de respuesta (ver Cuadro 2)

En síntesis, el trabajo consistió en preguntar y debatir con los asistentes a las diferentes TT.GG.NN que identificaran qué 
Cuadro 2. Modelo E.C.I.

\begin{tabular}{|c|c|c|c|c|c|c|}
\hline \multirow{2}{*}{\multicolumn{2}{|c|}{ Aspectos }} & \multirow{2}{*}{$\begin{array}{l}\text { Cuestiones: } \\
\text { valore en una escala de } 1 \text { (poco identificado) } \\
\text { a } 4 \text { (muy identificado) }\end{array}$} & \multicolumn{4}{|c|}{ Escala } \\
\hline & & & $\begin{array}{l}\text { Nada iden- } \\
\text { tificado }\end{array}$ & $\begin{array}{l}\text { Poco iden- } \\
\text { tificado }\end{array}$ & $\begin{array}{l}\text { Algo iden- } \\
\text { tificado }\end{array}$ & $\begin{array}{l}\text { Muy iden- } \\
\text { tificado }\end{array}$ \\
\hline \multicolumn{3}{|c|}{ Dimensión 1. Autoconocimiento Personal: Desde un punto de vista estrictamente personal: } & & & & \\
\hline 1. & $\begin{array}{l}\text { Conciencia emocional: reconocimiento de las } \\
\text { propias emociones y sus efectos. }\end{array}$ & $\begin{array}{l}\text { Soy consciente de los efectos que pueden tener } \\
\text { mis emociones }\end{array}$ & & & & \\
\hline 2. & $\begin{array}{l}\text { Autoevaluación: conocimiento de las fortale- } \\
\text { zas y limitaciones de uno mismo. }\end{array}$ & $\begin{array}{l}\text { Conozco mis propias fortalezas y limitaciones } \\
\text { personales }\end{array}$ & & & & \\
\hline 3. & $\begin{array}{l}\text { Autoconfianza: sentido de autoestima y de } \\
\text { las capacidades personales propias }\end{array}$ & Tengo confianza en mí mismo & & & & \\
\hline \multicolumn{7}{|c|}{ Dimensión 2. Autogestión: Desde una perspectiva emprendedora y en lo tocante a su perfil competencial } \\
\hline 4. & $\begin{array}{l}\text { Autocontrol emocional: contención de emo- } \\
\text { ciones e impulsos nocivos. }\end{array}$ & Ante una situación difícil me autocontrolo & & & & \\
\hline 5. & $\begin{array}{l}\text { Transparencia: integridad y actuación con- } \\
\text { gruentemente con los propios valores. }\end{array}$ & Actúo conforme a mis propios valores & & & & \\
\hline 6. & Adaptabilidad: flexibilidad ante los cambios. & Me adapto a los cambios & & & & \\
\hline 7. & $\begin{array}{l}\text { Logro: impulso por satisfacer y mejorar obje- } \\
\text { tivos propios de la excelencia. }\end{array}$ & Me impongo metas elevadas/exigentes & & & & \\
\hline 8. & $\begin{array}{l}\text { Iniciativa: disposición para actuar sobre las } \\
\text { oportunidades que se presentan }\end{array}$ & $\begin{array}{l}\text { Tengo iniciativa ante las oportunidades que se } \\
\text { presentan }\end{array}$ & & & & \\
\hline 9. & $\begin{array}{l}\text { Optimismo: capacidad para insistir en la con- } \\
\text { secución de objetivos pese a obstáculos. }\end{array}$ & Me considero una persona optimista & & & & \\
\hline \multicolumn{7}{|c|}{ Dimensión 3. Conciencia social: Desde una óptica relacionada con su entorno } \\
\hline 10. & $\begin{array}{l}\text { Empatía: capacidad para interiorizar senti- } \\
\text { mientos y perspectivas de los demás actuan- } \\
\text { do activamente en sus preocupaciones. }\end{array}$ & $\begin{array}{l}\text { Tengo capacidad para ponerme en "la piel de } \\
\text { otro" }\end{array}$ & & & & \\
\hline 11. & $\begin{array}{l}\text { Conciencia organizacional: capacidad para } \\
\text { interpretar corrientes emocionales de un gru- } \\
\text { po y relaciones de poder existentes. }\end{array}$ & $\begin{array}{l}\text { Me considero con capacidad para interpretar los } \\
\text { estados de ánimo de las personas de mi entorno }\end{array}$ & & & & \\
\hline 12. & $\begin{array}{l}\text { Orientación de servicio: anticipar, reconocer } \\
\text { y satisfacer las necesidades de los clientes. }\end{array}$ & $\begin{array}{l}\text { En mi trabajo me anticipo y reconozco las necesi- } \\
\text { dades que satisfacen a mis clientes }\end{array}$ & & & & \\
\hline \multicolumn{7}{|c|}{ Dimensión 4. Gestión de las Relaciones: Desde una visión emprendedora relacionada con la Gestión de los Recursos Humanos } \\
\hline 13. & $\begin{array}{l}\text { Desarrollo de personas: Detección de las } \\
\text { necesidades de desarrollo de las personas así } \\
\text { como el fortalecimiento de sus capacidades. }\end{array}$ & $\begin{array}{l}\text { Impulso el desarrollo personal y profesional de } \\
\text { mi equipo }\end{array}$ & & & & \\
\hline 14. & $\begin{array}{l}\text { Liderazgo inspirador: Inspirar a las personas } \\
\text { bajo un liderazgo efectivo. }\end{array}$ & $\begin{array}{l}\text { Con mi actuación motivo a las personas de mi } \\
\text { equipo }\end{array}$ & & & & \\
\hline 15. & $\begin{array}{l}\text { Catalizar el cambio: Iniciar o propulsar la } \\
\text { gestión del cambio. }\end{array}$ & Impulso el cambio en mi organización/empresa & & & & \\
\hline 16. & $\begin{array}{l}\text { Influencia: Implantar actividades orientadas a } \\
\text { la persuasión. }\end{array}$ & Soy persuasivo con las actividades que propongo & & & & \\
\hline 17. & $\begin{array}{l}\text { Gestión de conflictos: Negociación y resolu- } \\
\text { ción de desacuerdos. }\end{array}$ & $\begin{array}{l}\text { Gestiono los conflictos buscando una vía negocia- } \\
\text { da para conseguir un acuerdo }\end{array}$ & & & & \\
\hline 18. & $\begin{array}{l}\text { Trabajo en equipo y colaboración: Trabajar } \\
\text { con otros hacia metas compartida. Crear } \\
\text { sinergia de grupo en la búsqueda de objeti- } \\
\text { vos colectivos. }\end{array}$ & Trabajo en equipo & & & & \\
\hline
\end{tabular}

Fuente: elaboración propia a partir de Hay Group, McClelland Center for Research and Innovation. Prepared by Steven B. Wolff, DBA. Updated November 2005 Emotional Competence Inventory (ECI) Technical Manual. 
competencias de las 18 existentes favorecerían el emprendimiento en cada uno de los escenarios definidos con anterioridad.

\section{Resultados Comparativos}

Un somero análisis de las dimensiones señaladas en las diferentes TT.GG.NN (ver Cuadro 3) indica una importante coincidencia acerca de bajo qué contextos se identifican aspectos que contribuyen a dificultar el emprendimiento en personas con discapacidad.

En este sentido y en su conjunto, los aspectos donde se encuentran mayor número de limitaciones se localizan en la esfera del terreno personal y psicológico del individuo, seguido del espacio social-cultural y del institucional-legal. Los educativos-formativos y socioeconómicos pierden potencia explicativa y, por último, los relacionados con los aspectos físicos-accesibilidad no parecen ser tan relevantes con respecto a los otros.

Cuadro 3. Comparativa de escenarios por colectivos intervinientes en las TT.GG.NN.

\begin{tabular}{|l|c|c|}
\hline \multicolumn{1}{|c|}{ Escenarios } & $\begin{array}{c}\text { T.G.N. \% personas } \\
\text { discapacitadas }\end{array}$ & $\begin{array}{c}\text { T.G.N. \% técni- } \\
\text { cos AA.PP. }\end{array}$ \\
\hline Aspectos personales-psicológicos & 24,0 & 31,6 \\
\hline Aspectos socioculturales & 17,0 & 18,4 \\
\hline Aspectos institucionales-legales & 25,0 & 27,0 \\
\hline Aspectos educativos-formativos & 10,0 & 15,1 \\
\hline Aspectos socioeconómicos & 24,0 & 1,3 \\
\hline Aspectos físicos-accesibilidad & 0,0 & 6,6 \\
\hline Total & 100,0 & 100,0 \\
\hline
\end{tabular}

Fuente: Elaboración Propia.

6.1. Resultados desde la perspectiva de las Personas con Discapacidad

Profundizando un poco más en detalle en la T.G.N. realizada a personas con discapacidad sensorial y auditiva pueden distinguirse como principales rasgos:

Respecto a los factores limitadores del emprendimiento de las personas con discapacidad destacan los institucionales- legales $25 \%$, que representan el mayor peso explicativo en el conjunto de aspectos considerados. Los integrantes de la T.G.N. destacan las limitaciones que suponen para el emprendimiento cuestiones tales como la falta de vías de financiación adecuadas a las necesidades particulares de colectivos como el que nos ocupa. Junto a estos aspectos, también se denuncia la falta de oferta formativa para el emprendimiento. Otro grupo de factores serían las trabas burocráticas que acompañan a los procedimientos económicos y que, para estas personas, pueden suponer una dificultad adicional. Por último, se demanda mayor sensibilidad para la eliminación de barreras físicas, así como la dotación de medios humanos y técnicos especialmente pensados para el colectivo, como pueden ser traductores.

Los aspectos personales-psicológicos junto con los socioeconómicos ocupan un segundo lugar en la consideración de los participantes en esta T.G.N. con un peso del $24 \%$. En cuanto a los primeros, resultan relevantes las cuestiones derivadas de la propia discapacidad, que ralentizan aspectos como el aprendizaje. También destacan los derivados de la falta de empatía observada en la sociedad respecto a las dificultades con las que se tienen que enfrentar las personas discapacitadas en su día a día. Síntomas como la desmotivación, baja autoestima y poca confianza en sí mismos, son el resultado de la falta de comprensión percibida en el entorno.

En cuanto a los aspectos socioeconómicos (24\%), si bien no resultan específicos de las personas con discapacidad, sí tienen algunas particularidades que, en el caso de este colectivo vienen a dificultar un posible emprendimiento o su desarroIlo, en caso de estar iniciado. Destacan cuestiones tales como la falta de inversión económica en apoyo técnico y humano para personas discapacitadas, falta de inversión en aspectos directamente relacionados con las cuestiones empresariales y escasos o nulos incentivos fiscales.

Seguidos de los anteriores, y con un peso explicativo en la limitación al emprendimiento del $17 \%$ se han destacado los aspectos socioculturales. El común denominador a los límites señalados en este escenario es la falta de comprensión social sobre la discapacidad. Bajo esta premisa, los participantes relacionan cuestiones tan relevantes como las barreras en la comunicación social, la desconfianza que perciben en el resto de personas, la invisibilidad de la discapacidad y, con ella, de las dificultades que lleva aparejada cualquier actividad que afron$\tan$. A estos aspectos se unen otros que, aun siendo transversales, como la edad, en este caso constituyen dificultades adicionales en la inserción profesional y en el emprendimiento en particular.

Por último, en cuarto lugar, los participantes en esta T.G.N han identificado los aspectos educativo-formativos como elementos potencialmente limitadores del emprendimiento, con un peso explicativo significativamente menor que los anteriores (10\%). Entre las cuestiones mencionadas en este escenario, si bien existen elementos limitantes comunes para cualquier persona -con discapacidad o no-, como son la falta de conocimientos de marketing y otros específicos de la gestión empresarial, se enuncian otros directamente relacionados con los límites percibidos por este colectivo. Son cuestiones tales como una ausencia de formación específica para emprendedores 
con discapacidad u otros como la falta de recursos humanos de apoyo. Es el caso del colectivo con discapacidad auditiva usuario de la lengua de signos, que de forma reiterada denuncia la falta de intérpretes de esta forma de comunicación.

En términos competenciales (ver Cuadro 4) y, a partir de los resultados obtenidos, se desprende la existencia de un alto nivel de correspondencia entre las competencias de las cuatro dimensiones y la solución a las limitaciones planteadas a la hora de emprender. En concreto, las competencias correspondientes a la dimensión 2, "Autogestión", cubren todos los aspectos identificados en los 5 escenarios. Dentro de esta dimensión, adquieren especial relevancia, por su peso explicativo, las competencias siguientes: "Optimismo" (48,5\%); "Transparencia" (40\%) e "Iniciativa" (40\%). El hecho de cubrir todos los escenarios, a la vez que contener competencias con un mayor peso en la aportación de soluciones a los problemas planteados en dichos escenarios, da cuenta de la importancia de esta dimensión.

También las competencias de la dimensión 3 "Conciencia Social" servirían para dar respuesta a 4 de los 5 escenarios planteados (con la excepción del 1, que son los aspectos Institucionales). En esta dimensión, tendría un especial peso la competencia identificada como "Empatía" $(45,7 \%)$, un aspecto clave en los escenarios personal-psicológico y sociocultural.

Respecto a la dimensión 1, "Autoconocimiento", contiene competencias que responderían a varios de los escenarios considerados, con la excepción del institucional y el socioeconómico, con los que -según los participantes-, guarda una menor relación. En esta dimensión la "Conciencia emocional" y la "Autoconfianza" (ambas con un 40\%) son las destacadas por su relación con las cuestiones planteadas en los distintos escenarios.

Por último, en la dimensión 4, "Gestión de las relaciones", la competencia más relevante es el "Trabajo en equipo" (40\%), especialmente en el escenario educativo. Se trata de un resultado muy relevante, por lo que pueda suponer como orientación en la planificación de estrategias formativas. El resto de competencias de esta dimensión aparecen en menor medida relacionadas con la resolución de problemas planteados en los distintos escenarios; es el caso de la competencia "Catalizar el cambio" (28,6\%), una de las que obtiene un menor peso porcentual -junto con la "Orientación al servicio" (dimensión 3), de todas las competencias consideradas.

6.2. Resultados desde la perspectiva de los técnicos de la Administración

Desde la perspectiva de estos profesionales de la Administración, los factores limitadores del emprendimiento de las personas con discapacidad tienen que ver principalmente con variables de tipo personal-psicológico (31,6\%), que representan el mayor peso explicativo en el conjunto de aspectos considerados. Los integrantes de la T.G.N. destacan las limitaciones que suponen para el emprendimiento cuestiones tales como la baja autoestima y el miedo al fracaso, lo que puede ser resultado de la falta de comprensión percibida en el entorno. Es por esto que los integrantes de la T.G.N. también indican la necesidad de que la persona con discapacidad que decida emprender disponga de un entorno que le ofrezca apoyo. Por último, también se señalan las dificultades en el aprendizaje y la escasa motivación emprendedora.

Los aspectos institucionales ocupan un segundo lugar en la consideración de los participantes en esta T.G.N. con un peso del 27,0\%. Los integrantes de la T.G.N. destacan la ausencia de medidas de inclusión en asociaciones empresariales, la ausencia de cultura profesional junto con la falta de apoyo institucional. También destacan problemas administrativos serios como la incompatibilidad entre el desarrollo de una actividad laboral y el disponer de prestaciones sociales y exenciones fiscales. Finalmente, también se señalan otros aspectos como las dificultades que las personas con discapacidad encuentran en obtener asesoramiento y ligado a esto, la necesidad de más apoyos para el acceso a recursos económicos.

En cuanto a los aspectos socioculturales (18,4\%), destacan cuestiones tales como la escasa creatividad y formación educativa dirigida al emprendimiento, los miedos propios de las personas con discapacidad y, sobre todo, la existencia de un estigma social muy acentuado en relación a la actividad de emprendimiento de las personas con discapacidad.

En relación a los aspectos educativo-formativos (15,1\%), el común denominador a los límites señalados en este escenario es la falta de información y formación para el inicio de una actividad emprendedora, junto con el desconocimiento de la situación del mercado de trabajo y los nuevos yacimientos de empleo.

Seguidos de los anteriores, y con un peso explicativo en la limitación al emprendimiento del 6,6\% se han considerado los aspectos físicos-accesibilidad, que incluyen los problemas que encuentran las personas con discapacidad en la accesibilidad física a edificios e instalaciones.

Por último, en cuarto lugar, los participantes en esta T.G.N. han identificado los aspectos económicos como elementos potencialmente limitadores del emprendimiento, con un peso explicativo casi irrelevante, pues se queda en el 1,3\%. Entre las cuestiones mencionadas en este escenario, se habla de los problemas para obtener financiación y el miedo a la competencia.

En términos competenciales puede decirse que existe un significativo nivel de correspondencia entre las competencias de las cuatro dimensiones y la solución a las limitaciones planteadas a la hora de emprender. No obstante, son las competencias de la dimensión 1, "Autoconocimiento", las que dan solución a un menor número de escenarios, pues tanto la competencia 1 relacionada con la "Conciencia emocional" como la competencia 2 vinculada a la "Autoevaluación" no ofrecen soluciones más que al escenario de factores personales-psicológicos; sin embargo, es la competencia 3, "Autoconfianza" la que ofrece soluciones a más escenarios: personal-psicológico, 
Cuadro 4. Distribución de competencias por escenarios para el colectivo de personas con discapacidad (en \%)

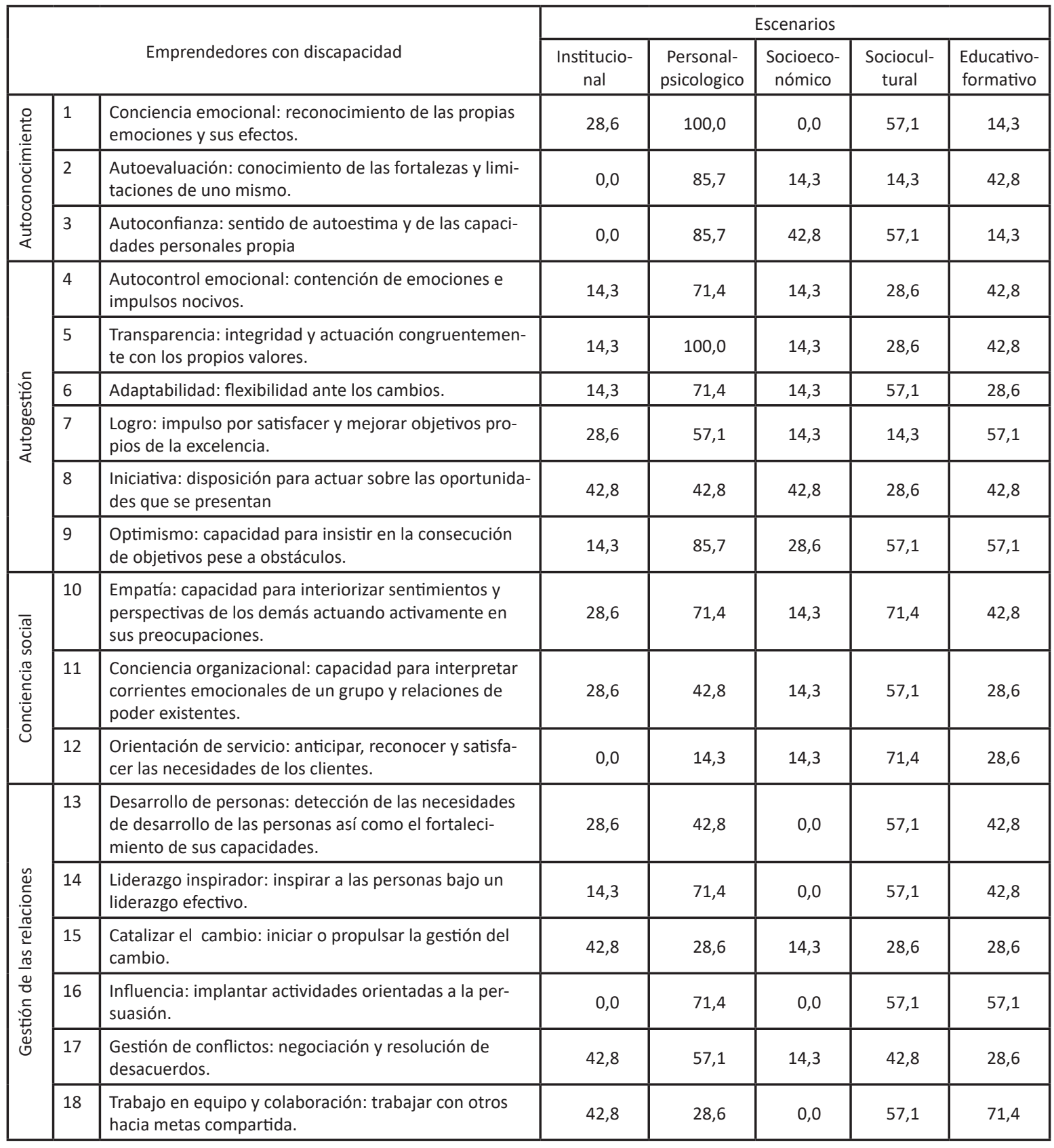

Fuente: elaboración propia. 
sociocultural, socioeconómico y educativo-formativo, teniendo un peso total del $26,2 \%$.

Por contra a lo visto anteriormente, la mayoría de las competencias de la dimensión 2, "Autogestión" cubren la mayor parte de los aspectos considerados en los cinco escenarios. Dentro de esta dimensión, son especialmente significativas las competencias relacionadas con la "Adaptabilidad" $(35,7 \%)$ y la "Iniciativa" (35,7\%), seguidas de la competencia vinculada al "Autocontrol emocional" (28,6\%).

En relación a la dimensión 3, "Conciencia social", todas sus competencias ofrecen respuesta a los cinco escenarios considerados, excepto las competencias "Conciencia organizacional" y "Orientación de servicio" que no ofrecen soluciones al escenario de aspectos físicos-accesibilidad. En esta dimensión, las tres competencias tienen pesos muy similares, que van desde el 33,3\% de la competencia "Empatía" hasta el 38,1\% de la competencia "Orientación de servicio", pasando por el 35,7\% de la "Conciencia organizacional".

Finalmente, en la dimensión 4, "Gestión de las relaciones", se comprueba que la mayor parte de las competencias tienen utilidad para resolver las dificultades planteadas en los escenarios considerados, aunque el escenario ligado a aspectos físicos-accesibilidad es aquel que tiene menos competencias capaces de solventar las dificultades que éste plantea ya que tan sólo la competencia ligada a "Gestión de conflictos" parece ofrecer utilidad. Por otro lado, esta dimensión contiene competencias con un mayor peso en la aportación de soluciones a los problemas planteados en los distintos escenarios. Destacan, especialmente, las competencias "Gestión de conflictos" (42,9\%), junto con el "Trabajo en equipo y colaboración" (33,3\%).

En relación a los escenarios, a la luz de los datos, parece que el relacionado con los aspectos físicos-accesibilidad es aquél en el que las competencias consideradas en las distintas dimensiones tienen una menor capacidad para solucionar los obstáculos o generados para el emprendimiento de personas con discapacidad. Lo contrario ocurre al observar la relación establecida entre las competencias de las diferentes dimensiones con el escenario personal-psicológico, lo que hace pensar que los problemas planteados por éste son más fácilmente solucionables con las competencias consideradas.

Por tanto, cabe destacar la importancia de la dimensión 4, "Gestión de las relaciones", para la resolución de los problemas planteados en todos los escenarios, y especialmente, en el institucional, el personal-psicológico", el sociocultural y el educativoformativo. En una posición similar se encuentran las dimensiones 2, "Autogestión" y 3, "Conciencia social". Por un lado, en la dimensión 2 hay que destacar su capacidad de resolución para los escenarios personal-psicológico, el sociocultural Social, el educativoformativo y el socioeconómico y, por otro lado, la dimensión 3 ofrece soluciones para todos los escenarios excepto el relacionado con los aspectos físico-accesibilidad. Finalmente, es la dimensión 1, "Autoconocimiento personal", la que tiene una menor relevancia.

\section{Conclusiones}

La perspectiva comparativa entre los colectivos implicados, confiere una especial riqueza a los resultados de este estudio. Si bien es importante señalar los aspectos coincidentes en el diagnóstico de los colectivos participantes -personas con discapacidad y técnicos de la Administración-, sobre los factores limitadores del emprendimiento en los primeros, no es menos significativo señalar las diferencias o intensificaciones de uno y otro colectivo en dichos aspectos.

En cuanto a las coincidencias, los resultados del análisis apuntan hacia los aspectos personales y psicológicos del individuo, seguidos de las cuestiones que tienen que ver con factores socioculturales y, en tercer lugar, por los de carácter institucional. Por el contrario, tanto para personas con discapacidad, como para técnicos, los elementos con menor peso explicativo sobre las limitaciones para emprender son los de índole educativa, así como los económicos. En último lugar se sitúan los relacionados con las limitaciones físicas, un aspecto reseñable y un síntoma, por una parte, de las destrezas adquiridas por las personas con discapacidad en este sentido y, por otra, de un avance en las cuestiones que facilitan la accesibilidad a espacios comunes.

Sin restar relevancia a las coincidencias, las diferencias halladas en el análisis comparativo adquieren especial interés. Respecto a éstas, es destacable que el primer factor limitador del emprendimiento de las personas con discapacidad son los factores institucionales-legales, mientras que para los técnicos de la Administración lo son las cuestiones de tipo personalpsicológico. Esta diferencia es sintomática, especialmente en lo que se refiere a los técnicos, a los que afecta especialmente la limitación de carácter institucional que señalan las personas con discapacidad. En cualquier caso, hay coincidencia en señalar en los primeros puestos estos dos aspectos (aunque en un orden diferente según el colectivo).

En tercera posición se observa también una cierta diferencia ya que, si bien para las personas con discapacidad, son limitadores significativos del emprendimiento la falta de incentivos económicos, los técnicos de la Administración conceden una mayor relevancia a cuestiones de carácter social, tales como la escasa creatividad y formación educativa dirigida al emprendimiento, los miedos propios de las personas con discapacidad y, sobre todo, la existencia de un estigma social.

Si bien es importante poner el acento en las limitaciones del emprendimiento para las personas con discapacidad, no lo es menos identificar las propuestas de solución a los obstáculos encontrados. En este sentido, la T.G.N., tomando como base el test $\mathrm{ECl}$, resulta muy reveladora y especialmente adecuada para llegar a soluciones a partir de la identificación de los problemas. Estas soluciones tienen en este estudio el denominador común de las competencias.

En este sentido, hay coincidencias en el diagnóstico de los dos colectivos respecto a la importancia de las competencias relacionadas con la dimensión "Autogestión", en concreto, se le concede relevancia a competencias tales como "Optimismo"; 
Cuadro 5. Distribución de competencias por escenarios para el colectivo de técnicos de la Administración (en \%)

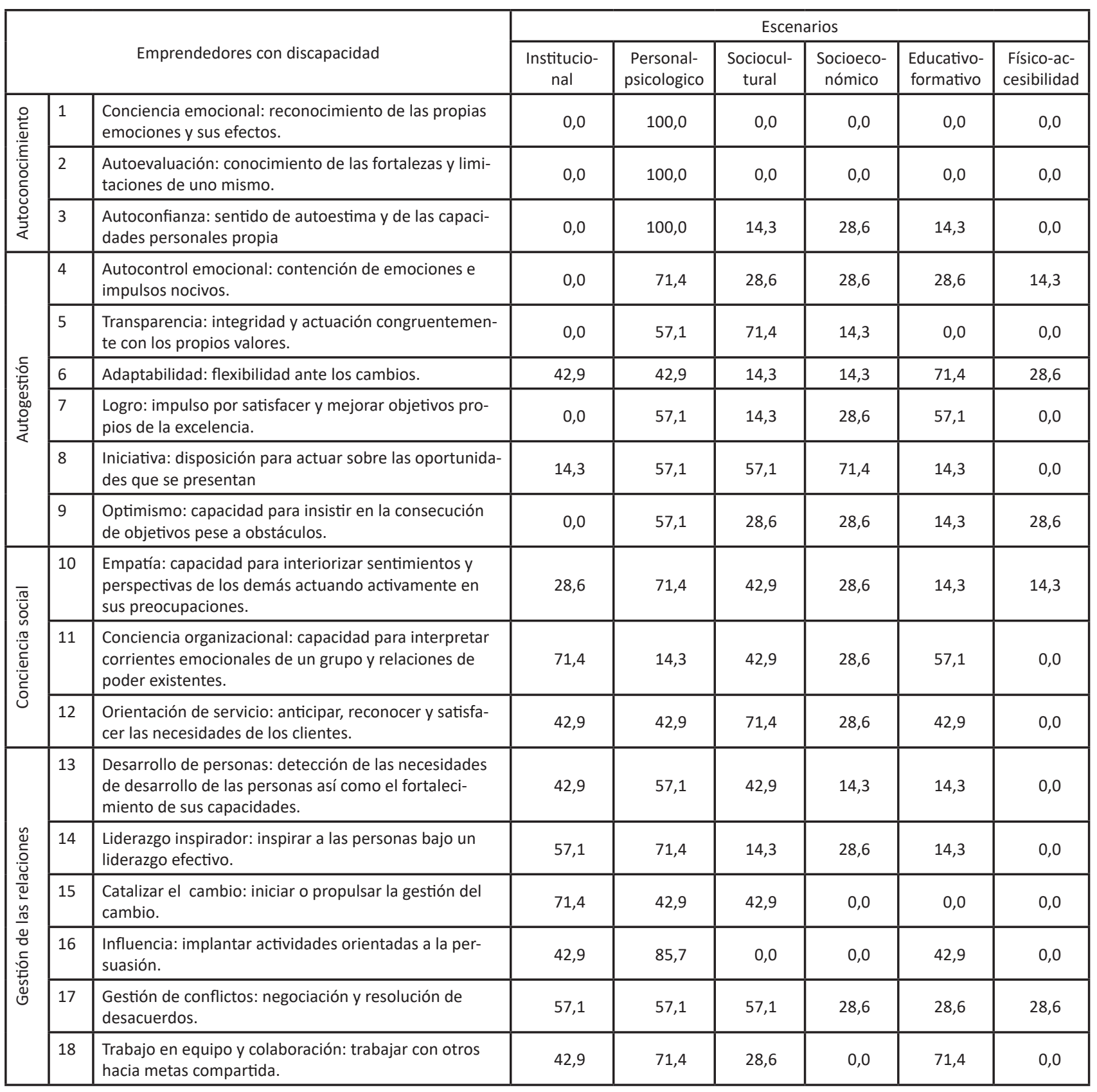

Fuente: elaboración propia. 
la Transparencia"; la "Iniciativa" (factores especialmente importantes para las personas con discapacidad en todos los frentes observados como barreras al emprendimiento, junto con la "Adaptabilidad" y la "Iniciativa" (observados por los técnicos de la Administración.

Unos y otros señalan con especial énfasis la importancia de la dimensión "Conciencia Social, especialmente el desarrollo de la "Empatía". Como también destacan en ambos casos la importancia de competencias en el ámbito de la "Gestión de las relaciones", de forma significativa la competencia de "Trabajo en equipos" y la capacidad para "Gestionar los conflictos".

Abundar en las competencias que ayudarían a hacer caer las barreras al emprendimiento en las personas con discapacidad es, sin duda, una apuesta por el futuro de esta actividad.
Bibliografía

BASCONES, L. M. y QUEZADA, M. Y. (2006): Daño cerebral sobrevenido en Castilla-La Mancha: realidad sociosanitaria, desafíos de apoyo e integración comunitaria, Toledo, FISLEM.

BLOOM, B. (1975): Evaluación del aprendizaje, Buenos Aires, Troquel.

BURCKLE, M. (2000): ECl and MBTI, Hay/McBer Research Report.

BYRNE, J. C. (2003): The role of emotional intelligence in predicting leadership and related work behavior. Hoboken: Stevens Institute of Technology, Technology Management.

CASADO, D. (1991): Panorámica de la discapacidad, Barcelona, Ed. Intress.

DE HARO, J. (2004): “¿Sabe alguien qué es una competencia?". Dirigir personas, 30, pp. 8-17.

DE LORENZO, R. (2003): E/ futuro de las personas con discapacidad en el mundo, Madrid, Fundación ONCE.

DELBECQ, A.; VAN DE VEN, A. y GUSTAFSON, D. (1975): Group techniques for program planning: A guide to nominal group and Delphi processes. Glenview, IL: Scott, Foresman.

DÍAZ, M. (1985): El asociacionismo de los minusválidos entre organización y movimiento social, Madrid, Universidad Complutense de Madrid.

DOBBIE, A.; RHODES, M.; TYSINGER. J. y FREEMAN, J. (2004). “Using a Modified Nominal Group Technique as a Curriculum Evaluation Tool", Family Medicine, 36 (6), pp. 402-406.

FERNÁNDEZ, E. (2005): Representaciones de la discapacidad intelectual. Tesis Doctoral, Universidad Complutense de Madrid. Recuperado de http://cisne.sim.ucm.es/search*spi/afernandez+cid/afernandez+cid/ $1 \% 2 \mathrm{C} 2 \% 2 \mathrm{C} 2 \% 2 \mathrm{CB} /$ frameset\&FF=afernandez+cid+enriquez+matilrd e\& $1 \% 2 \mathrm{C} 1 \% 2 \mathrm{C}]$.

GUTIÉRREZ, J. (2011): "Grupo de Discusión: ¿Prolongación, variación o ruptura con el focus group?", Cinta Moebio, 41, pp. 105-122.

HAY GROUP, McClelland Center for Research and Innovation (2005): Prepared by Steven B. Wolff, DBA, Updated November 2005, Emotional Competence Inventory (ECI) Technical Manual.

INE (2015): El Empleo de las Personas con Discapacidad. Explotación de la Encuesta de Población Activa y de la Base Estatal de Personas con Discapacidad. Año 2014. Notas de prensa. Recuperado de www.ine. es/prensa/prensa.htm.

INE (2008): Encuesta sobre Discapacidades, Deficiencias y Minusvalías 1986 (EDDM 1986).

INE (1999): Encuesta sobre Discapacidades, Deficiencias y Estado de Salud (EDDS 1999).

INE (2008): Encuesta sobre Discapacidades, Autonomía personal y situaciones de Dependencia 2008 (EDAD 2008). Recuperado de: http://www. ine.es/jaxi/menu.do?type=pcaxis\&path=/t15/p418\&file=inebase, .

JIMÉNEZ, A. (2007): “Conceptos y tipologías de la discapacidad: documentos y normativas de clasificación más relevantes". En De Lorenzo, R. y Pérez Bueno, L. (coords.), Tratado sobre discapacidad, Navarra, Thomson Reuters-Aranzadi, 177-205.

JIMÉNEZ A. (2005): "Bases demográficas: estimación, características y perfiles de las personas en situación de dependencia". Libro Blanco sobre la atención a las personas en situación de dependencia en España, Madrid, Ministerio de Trabajo y Asuntos Sociales.

JIMÉNEZ, A. (2002): Estudio sociodemográfico de la población con discapacidad en el Principado de Asturias, Oviedo, Fundación FASAD.

JIMÉNEZ, A. (2001): La imagen social de la discapacidad, en Jornadas sobre discapacidad y medios de comunicación, Cuenca, Asociación Roosevelt. Recuperado de http://usuarios.discapnet.es/Ajimenez/imagen/ imagensocial.htm.

JIMÉNEZ, A. (1998a):"El impacto de las nuevas tecnologías en el empleo de las personas con discapacidad". En: La situación del empleo de las per- 
sonas con discapacidad en España. Propuestas para su reactivación, Madrid, Escuela Libre Editorial.

JIMÉNEZ, A. (1998b): "Jóvenes con discapacidad. Características y necesidades", Juventud y Discapacidad, 43, pp. 13-21.

JIMÉNEZ, A. (1991): Infancia y discapacidad. Una aproximación cuantitativa. Primer Congreso Internacional Infancia y Discapacidad, vol. 2. Madrid, Ministerio de Asuntos Sociales.

JIMÉNEZ, A. (1992): Tipología y Prevalencia en España de las Deficiencias. Curso de Prevención de Deficiencias, Madrid, Real Patronato de Prevención y de Atención a Personas con Minusvalía.

JIMÉNEZ, A. y HUETE, A. (2003): Las Discapacidades en España: Datos estadísticos, Madrid, Real Patronato sobre Discapacidad.

JIMÉNEZ, A. y HUETE, A. (2002a): La Discapacidad en Cifras, Madrid, IMSERSO.

JIMÉNEZ, A. y HUETE, A. (2002b): La discriminación por motivos de discapacidad. Análisis de las respuestas recibidas al cuestionario sobre discriminación por motivos de discapacidad promovido por el CERMI Estatal, Madrid, CERMI.

LAWLER, E. (1994): “From Job - Based to Competence - Based Organizations", Journal of Organizational Behavior, 15 (1), pp. 3-15.

LE BOTERF, G.; VINCENT, F. y BARZUCCHETTI, S. (1993): Cómo gestionar la calidad de la formación, Barcelona, Gestión 2000.

MCCLELLAND, D. C. (1973): “Testing for competence rather than for "Intelligence '"', American Psychology, January, 28 (1), pp. 1-14.

OMS (1997). Clasificación Internacional de Deficiencias, Discapacidades y Minusvalías, Madrid, IMSERSO.

OMS (2001): Clasificación Internacional del Funcionamiento, de la Discapacidad y de la Salud, Madrid, Instituto de Migraciones y Servicios Sociales (IMSERSO).

PEREDA, S. y BERROCAL, F. (2001): Gestión de recursos humanos por competencias, Madrid, Centro de estudios Ramón Areces.

PÉREZ, L. (2004): El desmantelamiento de la discapacidad y otros escritos vacilantes, Barcelona, El Cobre.

RODRÍGUEZ, G. (2002): Apuntes sobre el Estado del Bienestar, Madrid, Universidad de Alcalá de Henares.

RODRÍGUEZ, G. y PÉREZ, M. (2004): Pobreza y exclusión social en el Principado de Asturias, Madrid, Consejo Superior de Investigaciones Científicas.

ROMAÑACH, J. (2002): Héroes y parias: la dignidad en la discapacidad. Recuperado de http://www.minusval2000.com/relaciones/vidalndependiente/heroes_y_parias.html.

ROMAÑACH, J. y PALACIOS, A. (2007): El modelo de la diversidad. La Bioética y los Derechos Humanos como herramientas para alcanzar la plena dignidad en la diversidad funcional, Valencia, Generalitat Valenciana ENIL.

RUIZ, J. (2012): "El grupo triangular: reflexiones metodológicas en torno a dos experiencias de investigación", Empiria Revista de Metodología de Ciencias Sociales, 24, pp. 141-162.

SALA, F. (2003): "Leadership in education: effective UK college Principals", Nonprofit Management \& Leadership, 14(2), pp. 171-189.

TUXWORTH, E. (1989): “Competence Based Education and Training: Background and Origins". En Burke, J. (ed.), Competency based education and training, London, The Falmer Press.

VÁZQUEZ FERREIRA, M. (2011): “Conformaciones, performaciones y transformaciones; herramientas transductivas de la contemporaneidad", Intersticios: Revista sociológica de pensamiento crítico, 5 (2), pp. 5-16.

VÁZQUEZ FERREIRA, M. (2010): "De la minus-valía a la diversidad funcional: un nuevo marco teórico-metodológico", Política y Sociedad, 47 (1), pp. 45-65.

VÁZQUEZ FERREIRA, M. (2008): "Una aproximación sociológica a la discapacidad desde el modelo social: apuntes caracteriológicos", REIS, 124, pp. 141-174.

VÁZQUEZ FERREIRA, M. (2007a): “Prácticas sociales, identidad y estratifi- cación: tres vértices de un hecho social, la discapacidad", Intersticios. Revista Sociológica de Pensamiento Crítico, 1 (2), 1-14,

VÁZQUEZ FERREIRA, M. (2007b): “Un nuevo concepto para la comprensión de la acción social: la transductividad creativa de las prácticas cotidianas", Intersticios. Revista Sociológica de Pensamiento Crítico, 1 (1), pp. 1-16.

VÁZQUEZ FERREIRA, M. (2005): "La reflexividad social transductiva: la construcción práctico-cognitiva de lo social y la sociología", Nómadas. Revista Crítica de Ciencias Sociales y Jurídicas, 11, pp. 287-303.

VAN DE VEN, A. y DELBECQ, A. (1972): "The nominal group as a research instrument for exploratory health studies", American journal of public health, 62 (3), 337-342.

WELPE, I., TUMASJAN, A., STICH, J., SPÖRRLE, M. y FÖRSTERLING, F. (2005): Emotional Intelligence and its consequences for occupational and life satisfactionEmotional Intelligence in the context of irrational beliefs. 47th conference of experimentally working psychologists, Lengerich, Pabst Science Publishers.

ZARCO, J. y GARCÍA DE LA CRUZ, J. J. (2004): El espejo social de la mujer con gran discapacidad, Madrid, Editorial Fundamentos. 